\title{
Influence of Silver Colloid on Fluorescein Spectral Properties
}

\author{
D. FrącKowiak ${ }^{a, *}$, A. BALter ${ }^{b}$, J. KAROLCZAK $^{c}$ \\ AND P. SIEJAK ${ }^{a}$ \\ ${ }^{a}$ Institute of Physics, Poznań University of Technology \\ Nieszawska 13A, 60-965 Poznań, Poland \\ ${ }^{b}$ Institute of Physics, N. Copernicus University \\ Grudziądzka 5/7, 87-100 Toruń, Poland \\ ${ }^{c}$ Faculty of Physics, A. Mickiewicz University \\ Umultowska 85, 61-614 Poznań, Poland
}

\begin{abstract}
In order to check if the observed earlier changes in fluorescence intensities of fluorescein solution due to the addition of colloidal suspension of silver are related to the generation of surface plasmon coupled emission, the steady state photothermal spectra and fluorescence decay times of such samples were measured. The Gaussian components of photoacoustic spectra for solutions with and without silver colloid addition were determined and compared with components of absorption and fluorescence excitation spectra of the same samples. The efficiencies of thermal and radiative deactivation of various samples were discussed. The fluorescence decay times measured at the wavelength of excitation not exhibiting the increase in photonic mode density depend only on the dye concentration. Decays exhibit changes as a result of silver addition with excitation in the region in which enhancement of fluorescence is observed. These changes are due not only to the change in the fluorescence yield generated by interactions with plasmons but also to those in thermal deactivation of the sample related to the colloid presence.
\end{abstract}

PACS numbers: 33.20.-t, 33.20.Kf, 33.50.--j

\section{Introduction}

Surface plasmon coupled emission (SPCE) effects have been recently applied in investigation of weakly fluorescent biological samples [1-4]. In SPCE effects the photonic mode density (PMD) of fluorophore located near to the metal surface increases. Such effect can be applied for example for determination of the presence

*corresponding author; e-mail: frackow@phys.put.poznan.pl 
and concentration of metals in river or lake water by comparing the fluorescence yields of the dyed samples with that of the samples of the same dye with additions of known amounts of metal. For ordered samples using polarized light, it is possible to draw information about the sample structure and aggregation [5].

In our earlier work [6] we have observed an increase in the yield of fluorescence of water solutions of fluorescein with addition of colloidal silver suspension. The nonradiative deactivation efficiency for dye exhibiting SPCE effect is usually the same in the presence and in the absence of metal in the sample [2,3]. We have to check if this condition is fulfilled for our fluorescein-Ag colloidal samples. If it is fulfilled, with increase in fluorescence yield the fluorescence decay time should be shorter because

$$
\tau=\left(\Gamma+k_{\mathrm{nr}}\right)^{-1}
$$

where $\tau$ - fluorescence decay time, $\Gamma$ - rate constant of fluorescence, and $k_{\mathrm{nr}}$ rate constant of nonradiative deactivation of excitation.

Fluorescein occurs in solutions in several ionic forms $[6,7]$. For such sample, the SPCE effect could be different for various forms and can be superimposed with effects generated by other mechanisms. The presence of microemulsions in the sample as well as the change in contents of various ionic forms of the dye can have influence on the yield of the fluorescence of fluorescein and the efficiency of nonradiative deactivation of the samples [1]. When nonradiative deactivation $\left(k_{\mathrm{nr}}\right)$ is changed, some other mechanisms must have the influence on the fluorescence decay time [7-9]. It is easier to observe the surface plasmon effects for a dye exhibiting low yield of radiative deactivation. In the case of fluorescein, exhibiting in solutions very high yield of fluorescence, such observations are more difficult, because global yield of deactivation cannot be higher than unity. The situation is complex because fluorescein can occur in several ionic forms and the presence of a colloid can change the contributions to the fluorescence of these forms [7]. Therefore, changes in the fluorescence intensity observed as a result of silver addition can be caused not only by the dye-plasmons interaction but also by other mechanisms. In order to establish the mechanisms responsible for the increase in the yield of fluorescence of fluorescein reported in our previous paper [6] and to support our supposition that the observed increase in the fluorescence yield could be due, at least partially, to SPCE [1], in this work we study thermal deactivation of the same systems as well as the fluorescence decay times measured at different wavelengths of excitation.

\section{Materials and methods}

Fluorescein was purchased from Sigma-Aldrich Chemie, Germany, colloidal silver particles were produced by Aurum Health Products, Ltd, Great Britain. All measured samples have $\mathrm{pH}=8$, therefore the contents of various ionic forms of the dye in various samples were similar. 
The photoacoustic spectra (PAS) were measured by the typical apparatus described in the Rosenzweig book [10] and our earlier papers [9]. It was a single beam photoacoustic spectrometer constructed in our laboratory [9]. The absorption spectra were taken using Cary 4000 (Varian), whereas the fluorescence excitation and emission spectra were measured by means of a Fluorescence Spectrophotometer F 4500 (Tokio, Hitachi, Japan). Fluorescence spectra were measured at front side configuration in which the reabsorption effect is not very high. But at high concentrations one cannot exclude some low influence of reabsorption on spectra.

Fluorescence decay times were measured by means of the apparatus constructed at the Institute of Physics of N. Copernicus University, Torun, Poland [11], or by the apparatus from the Physics Department of Adam Mickiewicz University in Poznań [12].

TABLE I

Thermal deactivation coefficients of samples with (TD) and without $\mathrm{Ag}\left(\mathrm{TD}_{0}\right)$, and $\mathrm{TD} / \mathrm{TD}_{0}$ calculated from the band areas and from intensities at $490 \mathrm{~nm}$. $C_{\mathrm{d}}$ and $C_{\mathrm{Ag}}$ - concentrations of dye and colloid silver suspension. TD and $\mathrm{TD}_{0}$ are in arbitrary units and accuracy of $\mathrm{TD}$ and $\mathrm{TD}_{0}-10 \%$.

\begin{tabular}{|c|c|c|c|c|c|c|c|c|}
\hline \multirow{3}{*}{$\begin{array}{c}C_{\mathrm{d}} \\
{[\mu \mathrm{M}]}\end{array}$} & \multirow{3}{*}{$\begin{array}{l}C_{\mathrm{Ag}} \\
{[\mu \mathrm{M}]}\end{array}$} & \multirow{3}{*}{$C_{\mathrm{Ag}} / C_{\mathrm{d}}$} & \multicolumn{6}{|c|}{$\mathrm{TD}$} \\
\hline & & & \multicolumn{3}{|c|}{ Band area } & \multicolumn{3}{|c|}{$\begin{array}{l}\text { Intensity at maximum } \\
\quad(\lambda=490 \mathrm{~nm})\end{array}$} \\
\hline & & & TD & $\mathrm{TD}_{0}$ & $\mathrm{TD} / \mathrm{TD}_{0}$ & $\mathrm{TD}$ & $\mathrm{TD}_{0}$ & $\mathrm{TD} / \mathrm{TD}_{0}$ \\
\hline 500 & 46 & 0.09 & 0.59 & 0.63 & $0.94 \pm 0.19$ & 0.52 & 0.54 & $0.97 \pm 0.20$ \\
\hline \multirow{2}{*}{100} & 46 & 0.46 & 1.36 & \multirow{2}{*}{2.23} & $0.61 \pm 0.12$ & 1.15 & \multirow{2}{*}{1.37} & $0.84 \pm 0.17$ \\
\hline & 83 & 0.83 & 1.20 & & $0.54 \pm 0.09$ & 0.99 & & $0.72 \pm 0.14$ \\
\hline
\end{tabular}

The PAS spectra were compared with the absorption and excitation spectra. All three types of spectra were decomposed into the Gaussian components belonging to various forms of the dye in order to check if PMD is the same for these forms. The thermal deactivation of fluorescein solutions (TD) with and without $\mathrm{Ag}\left(\mathrm{TD}_{0}\right)$ colloids were established and relative thermal deactivation $\mathrm{TD} / \mathrm{TD}_{0}$ was calculated. The calculations were done twice: taking into account whole areas of PAS and absorption spectra and taking values of these spectra at maxima at $490 \mathrm{~nm}$ (Table I). For a similar set of samples the relative yields of fluorescence were calculated.

\section{Results}

Absorption spectrum of $\mathrm{Ag}$ colloid at concentration about $10^{-4} \mathrm{M}$ is shown in Fig. 1. It is known that the position of the absorption of band of Ag colloid depends on the size and shape of particles [13]. As follows from the picture taken under an electron microscope of the colloids applied in our works [14], the colloid particles exhibit different sizes. As a result, broad flat absorption band in 


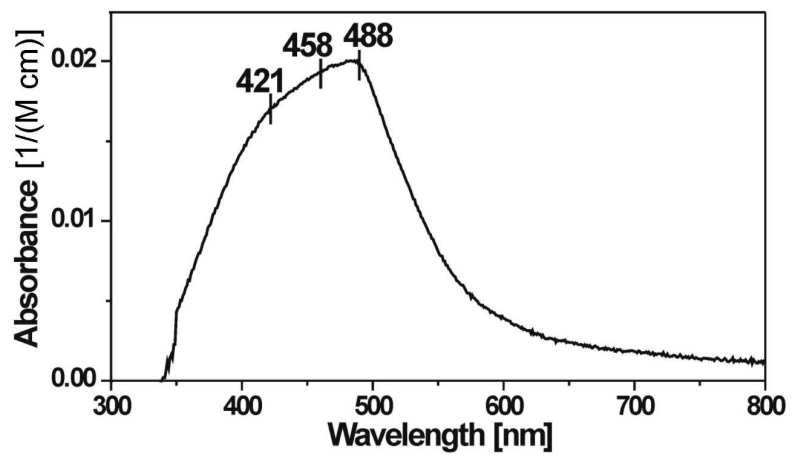

Fig. 1. Absorption spectrum of colloidal silver suspension in $1 \mathrm{~cm}$ cuvette. Silver concentration $9.3 \times 10^{-5} \mathrm{M}$.

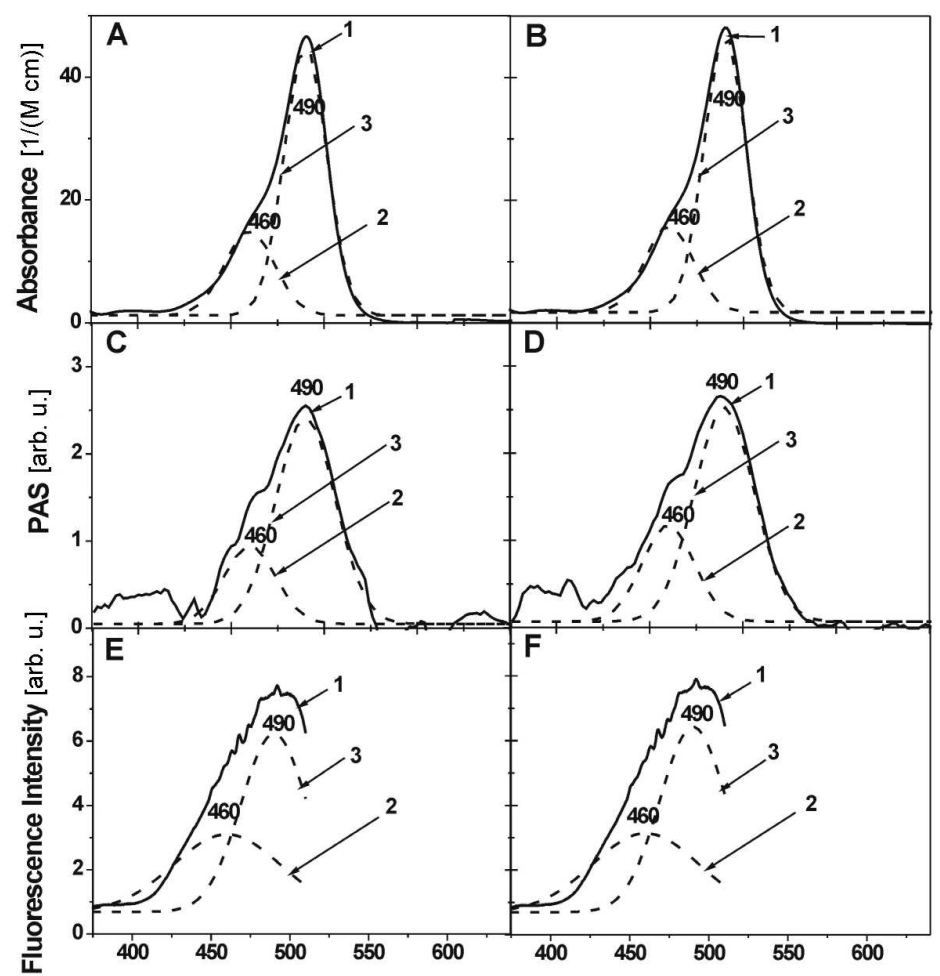

Fig. 2. Absorption ((A) and (B)), photoacoustic (PAS) ((C) and (D)), and fluorescence excitation $((\mathrm{E})$ and $(\mathrm{F}))$ spectra of investigated samples. $(\mathrm{A}),(\mathrm{C})$ and $(\mathrm{E})$ - sample without $\mathrm{Ag}$ colloidal suspension (dye concentration $\left.C_{\mathrm{d}}=5 \times 10^{-4} \mathrm{M}\right) ;(\mathrm{B}),(\mathrm{D})$ and (F) - sample with $\mathrm{Ag}$ colloidal suspension $\left(C_{\mathrm{d}}=5 \times 10^{-4} \mathrm{M}, \mathrm{Ag}\right.$ content $C_{\mathrm{Ag}}=$ $\left.4.6 \times 10^{-5} \mathrm{M}\right)$. Gaussian components - curves 2 and 3 . 
the region $350-600 \mathrm{~nm}$ (Fig. 1) is observed. It is not excluded that colloid particles of different size which contain different amounts of $\mathrm{Ag}$ nanocrystals could exhibit different efficiency of SPCE. The colloid absorption is much lower than that of fluorescein (Fig. 2), also the light scattering on the colloid particles in used experimental configuration can be neglected in analysis of the fluorescence spectra $[6,14]$.

The set of typical absorption, PAS and fluorescence excitation spectra measured for one of the used dye and colloid concentrations, decomposed into two Gaussian components, are shown in Fig. 2. One Gaussian component, with maximum at $460 \mathrm{~nm}$, is related predominantly to the acidic form of the dye, and the higher one, with maximum at about $490 \mathrm{~nm}$, is due predominantly to the alkaline form. As it follows from Fig. $2 \mathrm{C}$ and $\mathrm{D}$ the colloid addition changes the ratio of the area of those two components of TD. The analogous changes in the absorption (Fig. 2A and B) and fluorescence excitation spectra (Fig. 2E and F) are much lower.

On the basis of such spectra the relative values of thermal deactivations for the sample with colloid to that without colloid $\left(\mathrm{TD} / \mathrm{TD}_{0}\right.$, Table I) as well as the relative yields of fluorescence and fluorescence decay times (Table II) were calculated. The values of $\mathrm{TD} / \mathrm{TD}_{0}, \phi / \phi_{0}$ and $\tau / \tau_{0}$ in most cases are outside of

TABLE II

Fluorescence yield $(\phi)$ and fluorescence decay times $(\tau)$ of investigated samples. $\phi_{0}$ and $\tau_{0}$ - sample without $\mathrm{Ag}, \phi$ and $\tau$ - samples with Ag. $C_{\mathrm{d}}$ and $C_{\mathrm{Ag}}$ - concentrations of dye and colloid silver suspension. Accuracy of $\phi / \phi_{0}-0.05$, of $\tau$ and $\tau_{0}$ - 0.1 , and $\tau / \tau_{0}-4 \%$.

\begin{tabular}{|c|c|c|c|c|c|c|c|}
\hline $\begin{array}{c}\lambda_{\mathrm{ex}} \\
{[\mathrm{nm}]}\end{array}$ & $\begin{array}{c}C_{\mathrm{d}} \\
{[\mu \mathrm{M}]}\end{array}$ & $\begin{array}{c}C_{\mathrm{Ag}} \\
{[\mu \mathrm{M}]}\end{array}$ & $C_{\mathrm{Ag}} / C_{\mathrm{d}}$ & $\phi / \phi_{0}$ & $\begin{array}{c}\tau \\
{[\mathrm{ns}]}\end{array}$ & $\begin{array}{c}\tau_{0} \\
{[\mathrm{~ns}]}\end{array}$ & $\tau / \tau_{0}$ \\
\hline \multirow{4}{*}{488.0} & \multirow{2}{*}{100} & 46 & 0.46 & 1.04 & 5.41 & \multirow{2}{*}{5.62} & 0.96 \\
\hline & & 83 & 0.83 & 0.85 & 5.44 & & 0.98 \\
\hline & \multirow{2}{*}{50} & 46 & 0.92 & 1.42 & 4.86 & \multirow{2}{*}{5.12} & 0.95 \\
\hline & & 83 & 1.66 & 1.39 & 4.98 & & 0.97 \\
\hline \multirow{4}{*}{457.9} & \multirow{2}{*}{100} & 46 & 0.46 & 1.06 & 5.19 & \multirow{2}{*}{4.83} & 1.07 \\
\hline & & 83 & 0.83 & 0.85 & 5.46 & & 1.13 \\
\hline & \multirow{2}{*}{50} & 46 & 0.92 & 1.51 & 4.59 & \multirow{2}{*}{4.65} & 0.99 \\
\hline & & 83 & 1.66 & 1.46 & 4.60 & & 0.99 \\
\hline \multirow{3}{*}{421.0} & 100 & 83 & 0.83 & 0.94 & 5.09 & 5.09 & 1.00 \\
\hline & \multirow{2}{*}{50} & 46 & 0.92 & 0.99 & 4.73 & 4.73 & 1.00 \\
\hline & & 83 & 1.66 & 0.96 & 4.73 & 4.73 & 1.00 \\
\hline
\end{tabular}


accuracy of these values, showing the influence of silver colloid addition on those ratios. Relative thermal deactivation, in both cases, calculated from areas of bands and from their maxima, decreases with the increase in Ag amount (Table I). This decrease is stronger for the band areas than for the $490 \mathrm{~nm}$ maximum. The changes in thermal deactivation, according to formula (1), should have the influence on the fluorescence decay times (Table II) reducing their values with the increase in the fluorescence yield.

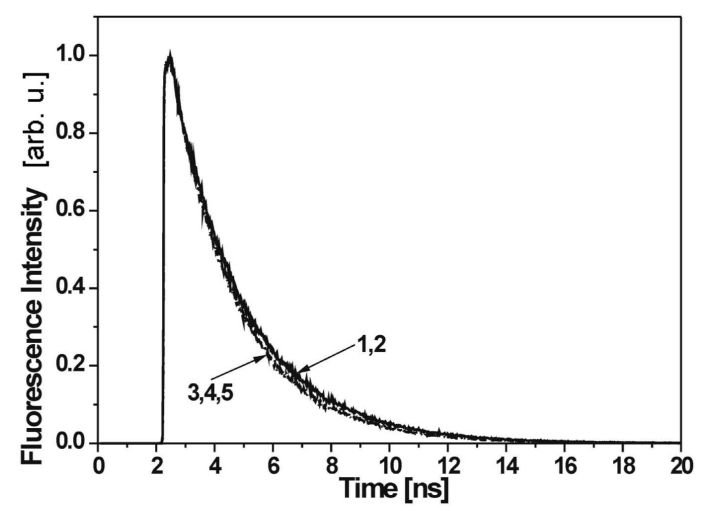

Fig. 3. Fluorescence decay curves of investigated samples. Curve $1-$ dye (dye concentration $\left.C_{\mathrm{d}}=1 \times 10^{-4} \mathrm{M}\right)$ without Ag colloid, curve $2-$ dye $\left(C_{\mathrm{d}}=1 \times 10^{-4} \mathrm{M}\right)$ with colloidal $\mathrm{Ag}$ addition $\left(\mathrm{Ag}\right.$ content $\left.C_{\mathrm{Ag}}=8.3 \times 10^{-5} \mathrm{M}\right)$, curve $3-$ dye $\left(C_{\mathrm{d}}=\right.$ $5 \times 10^{-5} \mathrm{M}$ ) without $\mathrm{Ag}$ colloid, curves 4 and 5 - dye with colloidal Ag addition (4$\left.C_{\mathrm{Ag}}=4.6 \times 10^{-5} \mathrm{M}, 5-C_{\mathrm{Ag}}=8.3 \times 10^{-5} \mathrm{M}\right)$. Excitation wavelength $-421 \mathrm{~nm}$.

As it follows from Table II, the decay times are almost independent of the amounts of the colloid added at the excitation at $421 \mathrm{~nm}$ at which the yield of fluorescence is not increasing. At this wavelength the colloid absorption is lower than at $460 \mathrm{~nm}$ and $490 \mathrm{~nm}$. In this short wavelengths region, the $\tau$ measured depends predominantly on the dye concentration, increasing slightly for higher amounts of fluorescein (Fig. 3). For $488 \mathrm{~nm}$ and $457.9 \mathrm{~nm}$ wavelengths of excitation the changes in $\tau / \tau_{0}$ value depend strongly on dye and colloid concentrations. For some samples the increase in $\phi / \phi_{0}$ is followed by the decrease in $\tau / \tau_{0}$, as it is expected from SPCE effect. For other combinations such regular dependence is not observed. Strong dependence on dye and colloid concentration suggests not uniform distribution of dye molecules in the sample. It is possible that concentration of dye near to the colloid surface can be higher than in the whole sample. At very high dye concentrations, the part of dye molecules may not be located near to the colloid surface and therefore they do not exhibit the increase in fluorescence intensity. The decrease in $\tau$ and increase in fluorescence yield strongly suggests the interactions of the dye with plasmons. 


\section{Conclusions}

The enhancement of the fluorescence intensity of fluorescein in the presence of a colloid containing Ag nanocrystals is not very high but measurable at some wavelengths and concentrations. The changes in the contents of various dye forms and in TD of the sample, both related to the colloid presence, make the quantitative analysis of plasmon influence complicated. It is possible that SPCE effect depends on the dimension of the colloidal particles, because the interactions between Ag nanocrystals and dye molecules could be not the same, for different colloidal particles. It is suggested by various enhancement of fluorescence and changes in fluorescence decay times observed at different excitation wavelengths, related to absorption of colloid of different sizes.

\section{Acknowledgments}

The authors were supported by the State Committee for Scientific Research (grant nr 2 P05D 046 30) coordinated by K. Marcinkowski University of Medical Sciences (Poznań, Poland) and by Poznań University of Technology (grant no. BW 62-207/06).

\section{References}

[1] J.R. Lakowicz, J. Malicka, I. Gryczynski, Z. Gryczynski, Ch.D. Geddes, J. Phys. D, Appl. Phys. 36, R240 (2003).

[2] J. Malicka, I. Gryczynski, Z. Gryczynski, J.R. Lakowicz, Anal. Chem. 75, 6629 (2003).

[3] H. Malak, in: The Teaching of the Physics in Technical Universities (Proc. Conf.), Ed. A. Gadomski, Academy of Technology and Agriculture, Bydgoszcz 2004, p. 15.

[4] I. Gryczynski, J. Malicka, K. Nowaczyk, Z. Gryczynski, J.R. Lakowicz, Thin Solid Films 510, 15 (2006).

[5] D. Frackowiak, L. Lorrain, D. Wrobel, R.M. Leblanc, Biochem. Biophys. Research Commun. 126, 254 (1985).

[6] P. Siejak, D. Frąckowiak, J. Phys. Chem. B 109, 14382 (2005).

[7] S. Biswas, S.Ch. Bhattacharya, P.K. Sen, S.P. Moulik, J. Photochem. Photobiol. A, Chem. 123, 121 (1999).

[8] J.R. Lakowicz, Principles of Fluorescence Spectroscopy, Kluwer Academic/Plenum Publishers, New York 1999.

[9] D. Frackowiak, S. Hotchandani, G. Bialek-Bylka, R.M. Leblanc, Photochem. Photobiol. 42, 567 (1985).

[10] A. Rosenzweig, Photoacoustics and Photoacoustic Spectroscopy, Wiley, New York 1980 .

[11] A. Balter, Nanosecond Adiabatic Processes of Excited Organic Particles in Solutions, N. Copernicus University Ed., Torun 1987 (in Polish). 
[12] J. Karolczak, D. Komar, J. Kubicki, T. Wróżowa, K. Dobek, B. Ciesielska, A. Maciejewski, Chem. Phys. Lett. 344, 154 (2001).

[13] Z. Gryczynski, J. Lukomska, J.R. Lakowicz, E.G. Matveeva, I. Gryczynski, Chem. Phys. Lett. 421, 189 (2006).

[14] P. Siejak, D. Frạckowiak, Plasmonics, submitted for publication. 